\title{
Wie erzielen wir Verbesserungen im klinischen Alltag?
}

\section{J. Zacher}

Qualitätsmanagement

Schlüssselvörter

Mortalitätskonferenz

Keywords

quality control

mortality conferences
Qualitätssicherung

\section{Einleitung \\ $\nabla$}

Mechanismen, die zur nachhaltigen Qualitätssicherung führen, sind in allen Bereichen des Lebens identisch. Qualität muss zuerst definiert, dann regelmäßig überprüft und gemessen werden. Letztlich müssen Anreizsysteme bestehen, die Qualität zu erhalten bzw. zu verbessern.

\section{Definition von Qualität \\ $\nabla$}

Im Helios-Konzern definieren 22 Fachgruppen medizinische Qualitätsparameter anhand der vorhandenen Literatur und eigener Expertise. Mitglieder sind die Chefärzte der jeweiligen medizinischen Spezialisten (z.B. Kardiologen). Qualitätsparameter werden anhand der Routinedaten, die den Krankenkassen zu Abrechnungszwecken übersandt werden, automatisch erzeugt und den medizinischen und betriebswirtschaftlichen Abteilungsleitern monatlich zur Verfügung gestellt.

\section{Erkennen von Qualitätsproblemen}

Helios arbeitet seit Jahren mit einem Set von Qualitätsindikatoren. Aus den monatlichen Routinedaten können Abweichungen unmittelbar, d.h. nur wenige Tage bis maximal 4 Wochen nach Auftreten erkannt werden. Dann arbeiten alle an der Behandlung des Patienten beteiligten Fachdisziplinen die Krankenakten auf und analysieren die abgelaufenen Prozesse hinsichtlich der rechtzeitigen und zielgerichteten Diagnostik und Therapie, der Einhaltung von Leitlinien etc. (Selbst-Review). Verbesserungspotentiale werden formuliert.

Institut

HELIOS Klinikum Berlin-Buch

Bibliografie

DOI $10.1055 / \mathrm{s}-0029-1242670$

Dtsch Med Wochenschr 2009;

134: S314 - (c) Georg Thieme

Verlag KG Stuttgart · New York .

ISSN 0012-0472

Korrespondenz

Prof. Dr. Josef Zacher

HELIOS Klinikum Berlin-Buch

Schwanebecker Chaussee 50

13125 Berlin

Tel. 030/9401-52300

eMail josef.zacher@

helios-kliniken.de

\section{Umgang mit identifizierten Qualitätsproblemen \\ $\nabla$}

Anhaltende Abweichungen einer Abteilung in einem der Qualitätsparameter führen zu einem Führungsgespräch mit der ökonomischen und medizinischen Leitung eines Krankenhauses. Auffällige Patientenverläufe, z.B. Todesfälle bei Eingriffen, bei denen der Laie erwartet, dass dabei niemand stirbt, wie elektiver Hüftgelenkersatz, komplizierte Patientenverläufe mit Beteiligung mehrerer Fachdisziplinen, die fatal verlaufen, oder Beinahe-Katastrophen werden in einer interdisziplinären Morbiditäts- und Mortalitätskonferenz (M\&M-Konferenz) vorge- stellt, analysiert, diskutiert und ein mögliches Verbesserungspotential erarbeitet. Nicht selten kann ein Verbesserungspotential, v.a. an Schnittstellen zwischen Abteilungen bzw. in klinikinternen Prozessen entdeckt und daraus Schlussfolgerungen gezogen werden, die in SOPs (standard operating procedures) münden. Mit deren Formulierung werden klinikinterne Experten beauftragt, die Texte im Intranet veröffentlicht und deren Umsetzung durch den Ärztlichen Direktor kontrolliert.

Die eigene Erfahrung mit der Einführung und Abhaltung von monatlichen M\&M-Konferenzen zeigt auf, dass klinikintern großes Interesse auf der Leitungsebene, aber auch auf allen weiteren Ebenen besteht, so dass diese CME-zertifizierten Konferenzen zu den am meisten akzeptierten Fortbildungsveranstaltungen zählen.

\section{Nachhaltigkeit}

Qualitätsprobleme, deren Ursache im Selbst-Review, in der Eigenanalyse und Auswertungsgeprächen zwischen Abteilungsleiter (Chefarzt) und Ärztlichem Direktor und Verwaltungsleitung identifiziert wurden, sind Gegenstand von Vereinbarungen zur Umsetzung von Verbesserungspotentialen.

\section{Zusammenfassung \\ $\nabla$}

Qualität kann auch in der Medizin nur erreicht werden, wenn sie definiert, regelmäßig kontrolliert und mit bewährten Maßnahmen zur Erzielung von Nachhaltigkeit verfolgt wird. Selbst-Reviews, Mortalitätskonferenzen und Transparenzmachung von Qualitätszielen sind geeignete Maßnahmen, um die erkannten Verbesserungspotentiale in den klinischen Alltag umzusetzen.

Autorenerklärung: Der Autor erklärt, dass keine relevanten finanziellen Verbindungen in Bezug auf dieses Manuskript bestehen.

Literatur

1 Mansky T et al. Ergebnisqualität sicher messen und aktiv verbessern - Erfahrungen. Medizinischer Jahresbericht 2006/2007. Berlin: HELIOS Kliniken Gruppe, 2008 